\title{
Uso de fauna y flora silvestre en la comunidad de Duyusupo y EI Jocote, Choluteca, Honduras
}

Use of wild fauna and flora in the community of Duyusupo and El Jocote, Choluteca, Honduras DOI 10.5377pc.v0i16.8097

Allison Flores, Rebeca Ortíz, Shasling Pacheco, Vivian Cabrera ${ }^{1}$

Luis Gutiérrez ${ }^{2}$

Nereyda Estrada ${ }^{3}$

\section{RESUMEN}

El aprovechamiento de la fauna y flora silvestre por parte de los seres humanos se remonta a sus orígenes mismos, sin embargo, su utilización produce un impacto en las mismas. Se entrevistó a pobladores de las comunidades de El Jocote y Duyusupo, ubicadas en el Área de Usos Múltiple la Botija dentro de la Reserva de Biósfera de San Marcos de Colón, para identificar las especies silvestres utilizadas. Con un diseño de muestreo no probabilístico por conveniencia, se aplicaron un total de 16 entrevistas semiestructuradas. Se identificó al menos ocho categorías de uso: consumo, cacería deportiva, importancia turística, mascotas, especies problemáticas, culturales, ornamentales, y medicinales. Dentro de los principales resultados se puede destacar el uso de 43 especies de fauna y 12 de flora silvestres. Las especies de fauna más utilizadas fueron los mamíferos (44.19\%), seguido de las aves (25.58\%). El 65\% de las especies que ellos identificaron, las consideraron como problemáticas y la categoría de consumo fue la segunda de mayor representatividad (28\%). En cuanto a la flora, el $62.5 \%$ se reporta para consumo, seguido por el uso de especies ornamentales (25\%). Se registro otras especies de importancia turística para los pobladores. Se sugiere realizar muestreos de campo para verificar las especies y evaluar estrategias de conservación de las mismas.

Palabras clave: Biosfera de San Marcos de Colón, La Botija, etnobiología.

\footnotetext{
1 Estudiante, Escuela de Biología, Facultad de Ciencias, UNAH. allison.flores@unah.hn, rebeca.ortiz @unah.hn, edda.pacheco@unah.hn, vivian.cabrera@unah.edu.hn

2 Asesor, Unidad de Proyectos, Sur en Acción-Ayuda en acción. Iguti25@yahoo.es

${ }^{3}$ Asesora, Profesora del Departamento de Biología, Escuela de Biología, Facultad de Ciencias, UNAH: nereyda.estrada@unah.edu.hn
} 


\section{ABSTRACT}

The exploitation of wild fauna and flora has been executed in a parallel way to the origins and development of human beings. However, its utilization produces an impact in these wild populations. This preliminary study was developed in El Jocote and Duyusupo communities located in the Multiple Area Use La Botija Mountain (AUMLB, by its spanish translation), in San Marcos de Colón Biosphere Reserve. This study makes a first approach with the population of these communities, for trying to identify the fauna and flora species, using as reference the common names that local population use for recognize them. And also for identifying the use that is given to wildlife in these communities. A non-probabilistic sampling design for convenience with 16 semi-structured interviews was implemented and developed in 6 hours. The species were distributed into 8 categories of use: consumption, sport hunting, tourist relevance, pets, problematic, cultural, ornamental, and medicinal. Among the main results we can highlight the use of 43 species of wild fauna and 12 species of flora. The species of fauna mostly used are mammals (44.19\%), followed by birds $(25.58 \%) .65 \%$ of the species were considered problematic, and consumption the second most represented $(28 \%)$. Regarding flora, $62.5 \%$ was considered as consumption, followed by ornamental $(25 \%)$. It should be noted that the inhabitants of both communities have identified some important species for them, with cultural and touristic potential, amongst others. However we need to continue this study for identifying the right species, their correct scientific name, and the use that is given by the local populations of these communities.

Keywords: San Marcos de Colon Biosphere, La Botija, ethnobiology. 


\section{INTRODUCCIÓN}

Las civilizaciones humanas a lo largo de la historia se han caracterizado por aprovechar las especies silvestres en un amplio espectro de usos acorde a sus necesidades, tales como fuentes alimenticias, medicinales, vestimenta, culturales, religiosas, simbólicas, intelectuales, ornamentales, como mascotas, entre otros (Monterubio, Lara, Piñera y Torres, 2005; Barrera-Bassols, 2005; González-Bocanegra, Romero-Berny, Escobar-Ocampo y García-Del Valle, 2011). Las condiciones sociales, culturales y económicas de una sociedad determinada, influyen de manera directa e indirecta en el grado de dependencia que una comunidad tendrá sobre la fauna silvestre (Pérez y Ojasti, 1996). En las zonas rurales el aprovechamiento de la fauna silvestre es, en mucho casos, una de las principales actividades humanas; donde la extracción de los recursos por un número importante de familias constituye una fuente primaria de consumo alimenticio de bajo costo (Challenger, 1998).

El uso de los recursos silvestres, además de estar asociado a los factores socioculturales, está determinado por la disponibilidad ecológica del mismo. Es así, que tanto la disponibilidad del recurso, el tiempo que demanda su preparación para consumo, o la edad de los recolectores, determinan el uso y frecuencia de las especies (Biurrun, Galetto, Anton, y Biurrun, 2007). Sin embargo, en el caso de las plantas medicinales, las exóticas cultivadas son más usadas que las silvestres, dada la facilidad a su acceso (Ladio y Lozada, 2004; Ochoa, Ladio y Lozada, 2010).

La fauna silvestre también representa amenazas a las comunidades humanas y sus cultivos, generando en muchos casos conflictos. Las plagas es una de las amenazas más comunes que afecta la producción de alimentos, por ejemplo en los cultivos de maíz, la gallina ciega del género Phyllophaga spp., se alimenta de las raíces, semillas y la base de los tallos, mientras que los adultos se alimentan del follaje de árboles y arbustos ocasionando defoliación severa (Potter, 1998; Escoto, 2004). Las especies silvestres representan un valor material cuantificable para la economía, desde al aporte proteico en la dieta, hasta la venta y uso de los subproductos como pieles, aceites y otros (Tratado de Cooperación Amazónica, 1995). Por lo anterior, identificar las especies utilizadas y cuantificar la frecuencia de uso de los recursos silvestres es relevante a la hora de diseñar políticas de conservación y manejo de áreas naturales (Ladio y Rapoport, 1999). El objetivo de la presente investigación fue la identificación preliminar de las especies de flora y fauna silvestre usada por las comunidades de Duyusupo y El Jocote en la Reserva de Biosfera de San Marcos de Colón, departamento de Choluteca. 


\section{MÉTODO}

\section{Área de estudio}

Las comunidades de Duyusupo y el Jocote se encuentran localizadas en el municipio de San Marcos de Colón, departamento de Choluteca. Este municipio se declaró Reserva de Biósfera San Marcos de Colon (RBSMC) el 14 de junio de 2017. La RBSMC tiene una extensión territorial de 57,810 ha., de clima tropical, con alturas entre 500 msnm y $1700 \mathrm{msnm}$. Las principales actividades productivas del municipio son la horticultura, fruticultura, floricultura, caficultura, ganadería y producción lechera, siendo reputada así mismo por sus productos de guarnicionería (UNESCO, 2017).

Dentro de la RBSMC se encuentra el Área de Usos Múltiples Montaña La Botija (AUMLB), con un área de 19,118 ha, se ubicada en las coordenadas $13^{\mathrm{a}} 15^{\prime} 58^{\prime \prime}$ y $13^{a} 23^{\prime} 18^{\prime \prime} \mathrm{N}$ y $86^{\mathrm{a}} 42^{\prime} 05^{\prime \prime}$ y $86^{\circ} 53^{\prime} 29^{\prime \prime} \mathrm{O}$. El AUMLB representa el $34 \%$ del área total de la Biosfera. Dentro del área se encuentran cinco aldeas y dieciocho comunidades con una población total estimada de 4,654 habitantes (ICF, 2009). El presente estudio se desarrolló en las comunidades de El Jocote y Duyusupo, dos de las dieciocho comunidades ubicadas dentro de la AUMLB (Figura 1).

Figura 1. Ubicación de las comunidades del Jocote y Duyusupo en el municipio de San Marcos de Colón, Choluteca. 2018. Fuente: ICF, SINIT, FACES. Elaboración: Rebeca Ortíz, 2018.

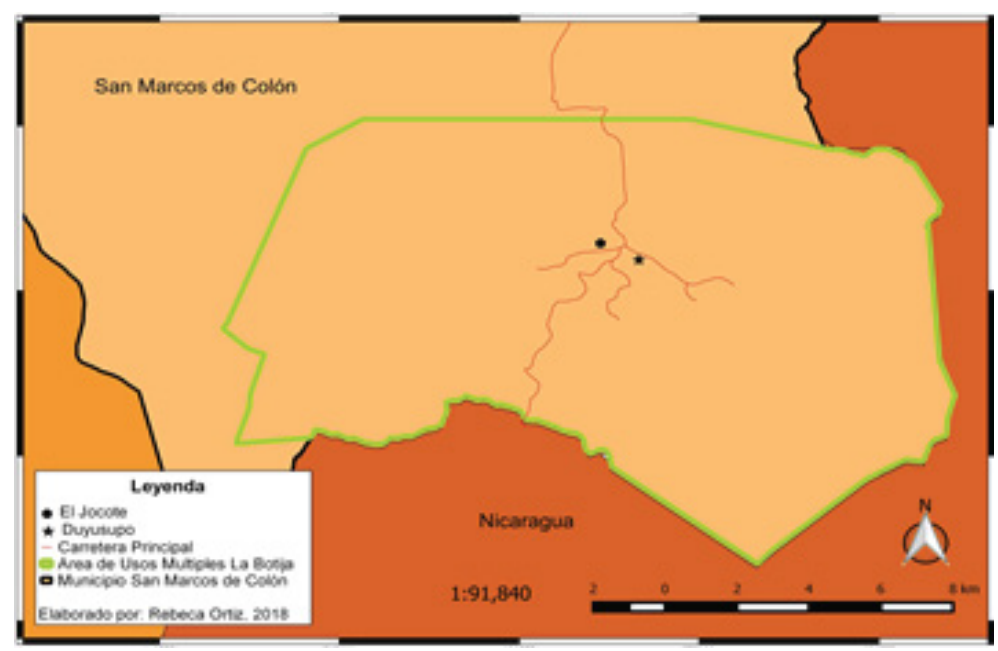




\section{Muestreo}

Se empleó un diseño de muestreo no probabilístico por conveniencia, cuya aplicación se emplea en los estudios exploratorios como el presente (Tamayo, 2001). Este tiene entre sus fundamentos la conveniente accesibilidad y proximidad de los sujetos al investigador (Otzen y Manterola, 2017). Además se empleó el método de bola de nieve de Goodman (1961), mediante entrevistas semiestructuradas, las cuales, por su carácter conversacional, facilita la comunicación entre los interlocutores (Ozonas y Pérez, 2005). Las entrevistas semiestructuradas permiten seleccionar los ejes a partir de los cuáles se generan las preguntas a realizar a la audiencia meta, las cuales incluyen un perfil socioeconómico, uso e identificación de las especies (Geilfus, 1997).

Las entrevistas se realizaron durante los días 19 y 20 de abril del 2018. Cada entrevista tuvo una duración promedio de 2.5 horas, aplicadas por cinco equipos conformados por dos entrevistadores. Se identificó a partir de las entrevistas, ocho categorías del uso de la flora y la fauna silvestre para la zona, definidas de la siguiente forma:

1. Consumo (C): Especies utilizadas con la finalidad de abastecer de carne, frutas, hojas y otros derivados para consumo propio y comercial (Miranda, 1993; Castañeda-Sifuentes y Albán-Castillo, 2016).

2. Cacería deportiva (CD): Esta actividad se practica como recreación, sin otra finalidad que su realización en sí, principalmente por sectores de la clase media y alta (Ojasti, 1993).

3. Importancia turística (T): Especies consideradas por los pobladores como un atractivo para turistas.

4. Mascotas (MA): Animales silvestres que los pobladores mantienen en cautiverio como animal de compañía.

5. Problemáticas $(\mathrm{P})$ : Aquellas que los pobladores consideran que ocasionan algún daño directo a sus cultivos, animales de crianza para consumo, animales domésticos, entre otros.

6. Culturales (CT): Incluye especies vinculadas a las creencias de la comunidad con fines mágicos, religiosos y ritualistas (Castañeda-Sifuentes y Albán-Castillo, 2016).

7. Ornamentales (O): Flora utilizada por los pobladores con fines decorativos para sus hogares.

8. Medicinales (M): Vinculadas al tratamiento de dolencias y percepciones patológicas sensibles al hombre (Castañeda-Sifuentes y Albán-Castillo, 2016). 
Para la identificación taxonómica preliminar de las especies se empleó los nombres comunes y las descripciones de morfología, coloración, tamaño, comportamiento, hábito y sitios de frecuencia, reportadas por los pobladores. En varios casos se utilizó imágenes de las especies para mostrarlas al entrevistado. Además, se verificó mediante una revisión bibliográfica la distribución y características de las especies registradas. Se empleó una modificación del Índice de Valor de Uso (IVU): IVU = $\Sigma$ $\mathrm{U} / \mathrm{n}$, donde $\mathrm{U}=$ número de citaciones por especie de cualquier categoría de uso y $\mathrm{n}=$ número de entrevistados (Phillips et al., 1994; Alves-Barbosa et al., 2010).

\section{RESULTADOS}

Se realizó un total de dieciséis entrevistas. De los entrevistados, nueve personas (56\%) son pobladores de la comunidad de El Jocote y siete $(44 \%)$ de la comunidad de Duyusupo. El $75 \%$ de los pobladores entrevistados son nativos de estas comunidades. El $56 \%$ de la muestra fueron hombres (nueve) y el $44 \%$ fueron mujeres (siete). Los entrevistados tuvieron un rango de edad entre 27 y 70 años, con un promedio de 50 años y una moda de 40 años. La muestra estuvo conformada principalmente por agricultores $(50 \%)$, ganaderos $(40 \%)$, pequeños comerciantes $(6 \%)$ y amas de casa $(4 \%)$.

En total, se identificó 42 posibles especies o grupos faunísticos en todas las categorías de uso. Los resultados sugieren que las especies a las que se les da mayor uso y representan mayor interés para los pobladores son los mamíferos con 18 especies (44\%), seguido de las aves con 11 especies (26\%), insectos con seis especies (14\%), cuatro especies de reptiles (9\%), dos especies de arácnidos (5\%) y una única especie de molusco (2\%) (Cuadro 1, 2, 3).

\section{Fauna}

El 28\% de las especies de fauna informadas por los pobladores están incluidas en los apéndices de la Convención sobre el Comercio Internacional de Especies Amenazadas de Fauna y Flora Silvestres (CITES). Siete especies se encuentran en el Apéndice III, cuatro especies en el Apéndice II y una especie en el Apéndice I (Cuadro 1, 2, 3). Además, el $56 \%$ de especies se incluyen en la Lista Roja de la Unión Internacional para la Conservación de la Naturaleza (UICN, 2018), en la categoría de preocupación menor con 22 especies, la guadaña (Cabassous centralis) y el venado colorado 
(Mazama temama) se encuentran en la categoría de datos insuficientes y la gallina de monte (Tinamus major) en la categoría de casi amenazada.

\section{Mamíferos}

El $52 \%$ de las especies de mamíferos se reportaron en la categoría problemática, es decir, organismos que los pobladores consideran que ocasionan algún daño directo a sus cultivos, animales de crianza para consumo, animales domésticos, entre otros (Cuadro 1). El guazalo (Didelphis sp.) fue la especie con mayor índice de valor de uso con un 0.75 , cuyas menciones fueron exclusivas para la categoría problemática por la frecuente incidencia en ataques de gallinas y alta abundancia en la zona. Seguido por el coyote (Canis latrans), murciélago vampiro (Desmodus rotundus) y gato de monte (Puma yagouaroundi), registradas por generar serios problemas por ataque a los animales de granja. Algunos entrevistados de mayor edad reportan registros de ataque a animales domésticos y de granja del león de montaña (Puma concolor). Estos registros datan de hace más de diez a quince años atrás por lo que se requiere una investigación más detallada para evidenciar su presencia en la zona.

La segunda categoría más reportada de las especies de fauna fue la de consumo (42\%). El venado (Odocoileus virginianus), el cusuco (Dasypus novemcinctus) y el conejo (Sylvilagus sp.) mostraron un alto IVU por consumo por su carne (Cuadro 1). Otras especies que son usadas también para consumo, pero que a su vez los pobladores identificaron como problemáticas por daños a los cultivos fueron los conejos (Sylvilagus sp.), guatusas (Dasyprocta punctata), ardillas (Sciurus sp.) y chanchos de monte (Tayassu tajacu). Se registró a la guadaña o pitero de diez bandas (Cabassous centralis) y el mono cara blanca (Cebus capucinus) como especies con potencial de atractivo turístico de la zona. En el caso de la guadaña también se reportó como peligroso el consumo de su carne por considerarla venenosa. En la categoría de uso para cacería deportiva, el venado cola blanca (Odocoileus virginianus) y el venado colorado (Mazama temama) fueron las únicas especies mencionadas. Además, algunos pobladores consideran que ambas especies también tienen un potencial de atractivo turístico en la zona. 


\section{Cuadro 1. Uso de los mamíferos identificados (taxa probable) por los pobladores de las comunidades El Jocote y Duyusupo 2018 (IVU= Índice de Valor de Uso, $C=$ consumo; $M=$ medicinal; $\mathrm{O}=$ ornamental; $\mathrm{P}=$ problemática).}

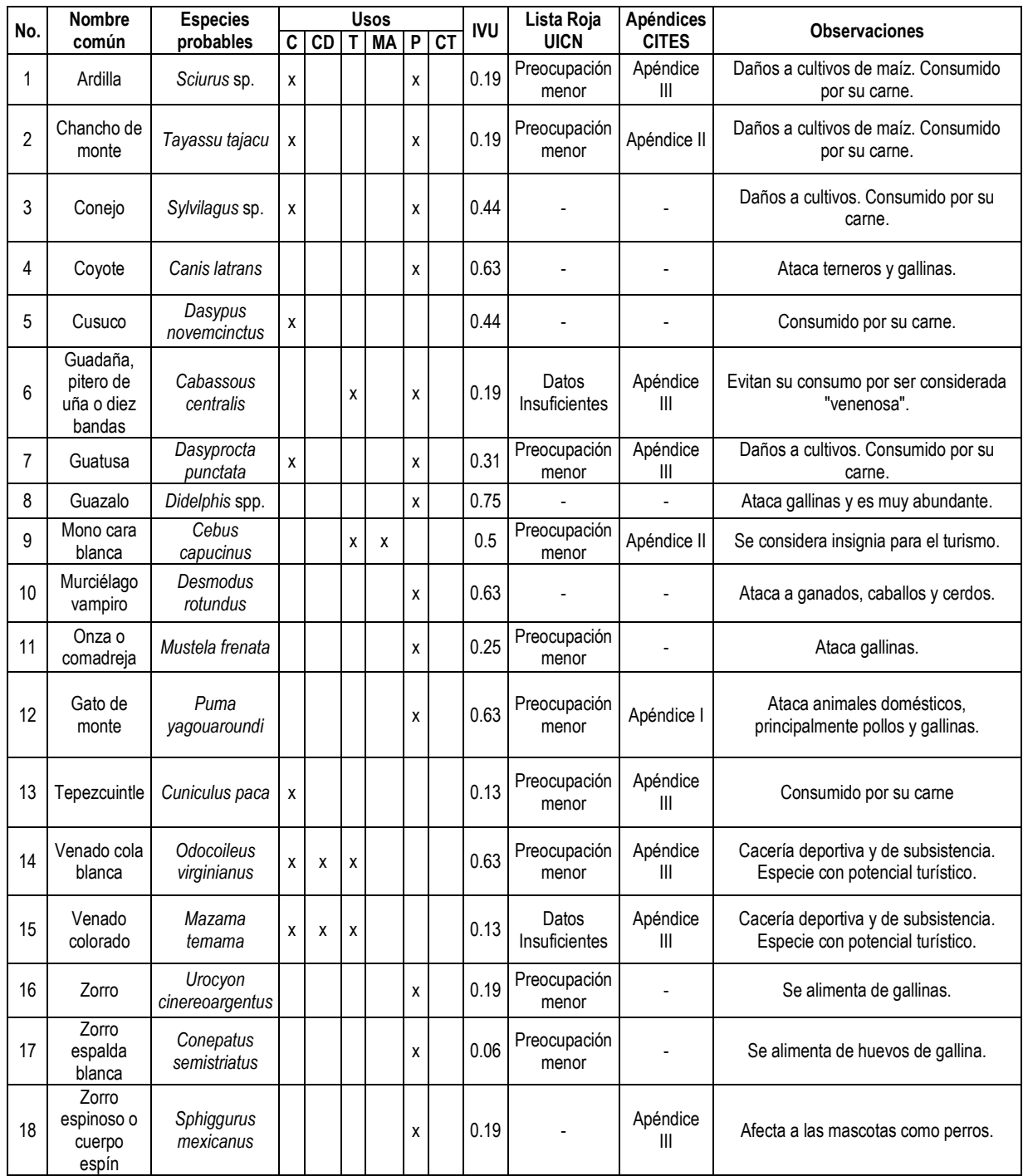

\section{Aves}

El segundo grupo más reportado por los entrevistados corresponde al de las aves. De las once especies mencionadas por los pobladores, cuatro son empleadas para consumo (36.36\%) (Cuadro 2). Según el IVU, la paloma ala blanca (Zenaida asiatica) con 0.5 es la de mayor demanda en términos de consumo, seguido de la gallina de 
monte (Tinamus major) con IVU 0.34. El psitácido Brotogeris jugularis (loro o sapoyol) se consume localmente pero también se usa como mascota en las mismas comunidades. La otra especie de psitácido mencionado fue Eupsittula canicularis (perico frente naranja) utilizado exclusivamente como mascota. Los pobladores identificaron el $36 \%$ de las especies de aves como problemáticas por la depredación y ataques a sus animales domésticos. La mayoría de estas especies pertenecen a la familia Accipitridae (gavilanes) con un IVU de 0.50. Por su parte, el zanate (Quiscalus mexicanus) con IVU 0.44, se reporta por afectar los cultivos al momento de la siembra. Se identificaron dos especies de importancia cultural, la lechuza (Tyto alba) y el guas o guaco (Herpetotheres cachinnans). La primera asociada con actividades de brujería y el segundo con el inicio de temporada lluviosa y sequías, así como el "anuncio" de la muerte de una persona.

\section{Cuadro 2. Uso de las aves identificados (taxa probable) por los pobladores en las comunidades de El Jocote y Duyusupo, 2018 (IVU= Índice de Valor de Uso, $\mathrm{C}=$ consumo; $\mathrm{M}=$ medicinal; $\mathrm{O}=$ ornamental; $\mathrm{P}=$ problemática).}

\begin{tabular}{|c|c|c|c|c|c|c|c|c|c|c|c|c|}
\hline \multirow{2}{*}{ No } & \multirow{2}{*}{$\begin{array}{l}\text { Nombre } \\
\text { común }\end{array}$} & \multirow{2}{*}{$\begin{array}{l}\text { Especies } \\
\text { probables }\end{array}$} & \multicolumn{6}{|c|}{ Usos } & \multirow[t]{2}{*}{ IVU } & \multirow{2}{*}{$\begin{array}{l}\text { Lista Roja } \\
\text { UICN }\end{array}$} & \multirow{2}{*}{$\begin{array}{l}\text { Apéndic } \\
\text { es } \\
\text { CITES }\end{array}$} & \multirow{2}{*}{ Observaciones } \\
\hline & & & C & $C D$ & $\mathrm{~T}$ & MA & $P$ & $\mathrm{CT}$ & & & & \\
\hline 1 & Codorniz & $\begin{array}{l}\text { Colinus } \\
\text { cristatus }\end{array}$ & $x$ & & & & & & 0.06 & $\begin{array}{l}\text { Preocupaci } \\
\text { ón menor }\end{array}$ & - & Consumido por su carne. \\
\hline 2 & Estiquirin & $\begin{array}{l}\text { Bubo } \\
\text { virginianus }\end{array}$ & & & & & $x$ & & 0.19 & $\begin{array}{l}\text { Preocupaci } \\
\text { ón menor }\end{array}$ & - & Amenaza a las mascotas. \\
\hline 3 & $\begin{array}{l}\text { Gallina } \\
\text { de monte }\end{array}$ & $\begin{array}{l}\text { Tinamus } \\
\text { major }\end{array}$ & $x$ & & & & & & 0.34 & $\begin{array}{c}\text { Casi } \\
\text { amenazado }\end{array}$ & - & $\begin{array}{l}\text { Consumida por su carne y huevos. } \\
\text { Utilizadas para exhibición. }\end{array}$ \\
\hline 4 & Gavilán & Accipitridae & & & & & $x$ & & 0.5 & - & - & $\begin{array}{l}\text { Amenaza por alimentarse de } \\
\text { animales domésticos. }\end{array}$ \\
\hline 5 & $\begin{array}{l}\text { Guas o } \\
\text { Guaco }\end{array}$ & $\begin{array}{l}\text { Herpetotheres } \\
\text { cachinnans }\end{array}$ & & & & & & $x$ & 0.13 & $\begin{array}{l}\text { Preocupaci } \\
\text { ón menor }\end{array}$ & - & $\begin{array}{l}\text { "Anuncia la temporada de lluvia y } \\
\text { sequía", y su canto anuncia la } \\
\text { muerte de un poblador. }\end{array}$ \\
\hline 6 & Lechuza & Tyto alba & & & & & & $x$ & 0.25 & $\begin{array}{l}\text { Preocupaci } \\
\text { ón menor }\end{array}$ & - & $\begin{array}{c}\text { Considerada como un "ave mala", } \\
\text { relacionada con prácticas de } \\
\text { brujería. }\end{array}$ \\
\hline 7 & $\begin{array}{l}\text { Loros o } \\
\text { sapoyole } \\
\text { S }\end{array}$ & $\begin{array}{l}\text { Brotogeris } \\
\text { jugularis }\end{array}$ & $x$ & & & $x$ & & & 0.19 & $\begin{array}{l}\text { Preocupaci } \\
\text { ón menor }\end{array}$ & $\begin{array}{c}\text { Apéndice } \\
\text { II }\end{array}$ & $\begin{array}{c}\text { Capturados para comercializarlos o } \\
\text { mascotas. }\end{array}$ \\
\hline 8 & $\begin{array}{l}\text { Paloma } \\
\text { ala } \\
\text { blanca }\end{array}$ & $\begin{array}{l}\text { Zenaida } \\
\text { asiatica }\end{array}$ & $x$ & & & & & & 0.5 & $\begin{array}{l}\text { Preocupaci } \\
\text { ón menor }\end{array}$ & - & Consumido por su carne. \\
\hline 9 & Pericos & $\begin{array}{l}\text { Eupsittula } \\
\text { canicularis }\end{array}$ & & & & $x$ & & & 0.25 & $\begin{array}{l}\text { Preocupaci } \\
\text { ón menor }\end{array}$ & $\underset{\text { II }}{\text { Apéndice }}$ & $\begin{array}{c}\text { Mascota muy frecuente en las } \\
\text { viviendas de los pobladores de la } \\
\text { zona. }\end{array}$ \\
\hline 10 & Urraca & $\begin{array}{l}\text { Calocitta } \\
\text { formosa }\end{array}$ & & & & & $x$ & & 0.19 & $\begin{array}{l}\text { Preocupaci } \\
\text { ón menor }\end{array}$ & - & $\begin{array}{l}\text { Atacar los huevos de aves } \\
\text { domésticas. }\end{array}$ \\
\hline 11 & Zanate & $\begin{array}{l}\text { Quiscalus } \\
\text { mexicanus }\end{array}$ & & & & & $x$ & & 0.44 & $\begin{array}{l}\text { Preocupaci } \\
\text { ón menor }\end{array}$ & - & Daños a cultivos \\
\hline
\end{tabular}




\section{Reptiles}

Los pobladores mencionaron únicamente cuatro especies de reptiles en la categoría de organismos problemáticos. El cascabel (Crotalus simus) y los corales (Micrurus spp.) con un IVU 0.19 son temidos por los pobladores por su alta peligrosidad por mordeduras y envenenamiento. La cascabel y la mica (Spilotes pullatus) registraron el mayor IVU con 0.31, ambas por amenazas a ataques a las personas y sus animales domésticos. En el caso de la boa (Boa imperator) se reporta con un IVU de 0.25 , principalmente por ataques a aves de corral.

\section{Insectos}

En el caso de los insectos, se consultó solamente por especies problemáticas. Dentro de los principales daños reportados se encuentran a los cultivos, ganado, flora silvestre y a la salud humana (Cuadro 3 ). Una de las principales plagas reportadas es la gallina ciega (IVU 0.44), una larva de las familias Scarabaeidae y Melolonthidae (Morón Ríos, 1986), posiblemente de los géneros Cyclocephala, Ataenius, Cotinis, Popillia, Anomala y Phyllophaga. Las especies del género Phyllophaga representan una de las principales plagas de América Latina (Potter, 1998). La langosta ocupa el segundo lugar en cuanto al IVU (0.25), en Centroamérica existen cuatro especies del género Schistocerca, sin embargo, únicamente Schistocerca piceifrons piceifrons tiene las aptitudes para convertirse en plaga de cultivos (SENASICA-DGSV, 2016). Según los pobladores tanto la gallina ciega como la langosta afectan principalmente los cultivos de frijol y maíz, además de los cultivos de hortalizas, leguminosas, cítricos, caña de azúcar, café, entre otros.

De los insectos mencionados, el tórsalo (Dermatobia sp.) ocupa el tercer lugar IVU de 0.19 , los pobladores consideran que afecta al ganado principalmente. Esta especie no es considerada plaga por la baja cantidad de individuos y lo poco perjudicial que es, pues no implica muerte del organismo ni representa daños económicos graves; sin embargo, genera intranquilidad, estrés y malestar en los animales (Cruz, 2005). Se mencionó a la chinche picuda (Triatoma dimidiata), vector del mal de chagas causada por el protista Tripanosoma cruzi, sin embargo, su mención fue poco frecuente entre los entrevistados. 
Cuadro 3. Insectos identificados (taxa probable) por los pobladores en las comunidades de El Jocote y Duyusupo, 2018 (IVU= Índice de Valor de Uso, $\mathrm{C}=$ consumo; $\mathrm{M}=$ medicinal; $\mathrm{O}=$ ornamental; $\mathrm{P}=$ problemática).

\begin{tabular}{|c|l|l|c|l|}
\hline No & \multicolumn{1}{|c|}{$\begin{array}{c}\text { Nombre } \\
\text { común }\end{array}$} & $\begin{array}{c}\text { Familia o } \\
\text { especie }\end{array}$ & IVU & \multicolumn{1}{|c|}{ Observaciones } \\
\hline 1 & Langosta & $\begin{array}{l}\text { Schistocerca } \\
\text { spp. }\end{array}$ & 0.25 & $\begin{array}{l}\text { Afecta los cultivos de caña de azúcar, frijol, hortalizas, cítricos, } \\
\text { gramíneas, y leguminosas, como macuelizo y el carbón. }\end{array}$ \\
\hline 2 & $\begin{array}{l}\text { Gallina } \\
\text { ciega }\end{array}$ & Scarabaeidae & 0.44 & Causa daño a los cultivos a nivel de raíz. \\
\hline 3 & Tórsalo & Dermatobia spp. & 0.19 & Ocasiona daño a cualquier vertebrado. \\
\hline 4 & Chicharra & Empoasca spp. & 0.13 & Afecta cultivos principalmente de frijol. \\
\hline 5 & $\begin{array}{l}\text { Mosca } \\
\text { blanca }\end{array}$ & Bemisia spp. & 0.06 & Afecta cultivos de frijol. \\
\hline 6 & $\begin{array}{l}\text { Chinche } \\
\text { picuda }\end{array}$ & $\begin{array}{l}\text { Triatoma } \\
\text { dimidiata }\end{array}$ & 0.06 & $\begin{array}{l}\text { Vector de Trypanosoma cruzi, ocasiona la enfermedad del mal de } \\
\text { Chagas. }\end{array}$ \\
\hline
\end{tabular}

\section{Arácnidos y Moluscos}

Se mencionó por los pobladores dos morfoespecies de arácnidos, categorizados como problemáticos, ambas con IVU de 0.25 , las garrapatas (Ixodoidea) y araña "orina caballos" (Brachypelma sp., o Aphonopelma sp.). Las garrapatas parasitan diferentes especies de mamíferos (incluido el hombre), aves y reptiles (Estrada, 2015). Únicamente se identificó una morfoespecie de molusco considerada problemática por los pobladores, reportando un IVU de 0.25. Se presume según distribución, hábito y estudios realizados para plagas del frijol, la babosa o ligosa (Sarasinula plebeia) es la que potencialmente se encuentra presente en la Montaña La Botija (Andrews y Dundee, 1987; Caballero et al., 1991).

\section{Flora}

Se registró un total de 12 especies vegetales, pertenecientes a 10 Familias y 10 géneros con cuatro usos diferentes (Cuadro 4). En el caso de flora, tres posibles especies (25\%) se encuentran catalogadas en CITES, Hylocereus spp. y la familia Orchidaceae en el Apéndice II y únicamente Cedrela odorata en el Apéndice III. También se reportaron tres especies (25\%) dentro de la lista roja de UICN, Cedrela odorata catalogada como vulnerable y dos especies Pinus oocarpa y Pinus maximinoi enlistada como casi amenazadas.

La mayoría de las plantas fueron reportadas en la categoría de consumo (69\%), principalmente en el aprovechamiento para madera de construcción y leña. La especie con mayor IVU 0.75 fue el roble (Quercus spp.), seguido por pinos (Pinus oocarpa y Pinus maximinoi) con un IVU de 0.63 . En menor medida el cedro (Cedrela odorata) y quebracho (Lysiloma divaricatum). Se incluye dentro de la categoría de consumo 
los especies con beneficios proteicos que los pobladores obtienen por el consumo directo de diferentes órganos vegetales, entre estas la flor de izote (Yucca guatemalensis), tallos y frutos de pitaya (Hylocereus spp.) y frutos de moras silvestres (Rubus ulmifolius). Algunas personas reportaron a los cactus como especies problemáticas, pues su presencia en zonas ganaderas ocasiona daños físicos al ganado debido a las espinas que la planta posee.

Se registró el helecho Phlebodium spp., comúnmente conocido como calaguala, dentro de la categoría de medicinales por sus cualidades para contrarrestar patologías renales. La flora extraída durante todo el año del medio silvestre con fines ornamentales se encuentra representada por orquídeas IVU de 0.06, o bien se extraen para festividades específicas como las celebraciones decembrinas, época en la que se utiliza barba de viejo (Tillandsia usneoides) y musgo como parte de las decoraciones distintivas de estas fechas.

Cuadro 4. Uso de la flora identificada (taxón probable) por los pobladores de comunidades de El Jocote y Duyusupo, 2018 (IVU= Índice de Valor de Uso, $\mathrm{C}=$ consumo; $\mathrm{M}=$ medicinal; $\mathrm{O}=$ ornamental; $\mathrm{P}=$ problemática).

\begin{tabular}{|c|c|c|c|c|c|c|c|c|c|c|}
\hline \multirow[t]{2}{*}{ No. } & \multirow{2}{*}{$\begin{array}{l}\text { Nombre } \\
\text { común }\end{array}$} & \multirow{2}{*}{$\begin{array}{c}\text { Nombre científico } \\
\text { probable }\end{array}$} & \multicolumn{4}{|c|}{ Usos } & \multirow[t]{2}{*}{ IVU } & \multirow{2}{*}{$\begin{array}{l}\text { Lista Roja } \\
\text { UICN }\end{array}$} & \multirow{2}{*}{$\begin{array}{l}\text { Apéndices } \\
\text { CITES }\end{array}$} & \multirow[t]{2}{*}{ Observaciones } \\
\hline & & & $\mathrm{C}$ & $M$ & 0 & $P$ & & & & \\
\hline 1 & Musgo & Bryophyta & & & $x$ & & 0.06 & - & - & Decoración fiestas navideñas. \\
\hline 2 & $\begin{array}{l}\text { Barba de } \\
\text { viejo }\end{array}$ & $\begin{array}{c}\text { Tillandsia usneoides } \\
\text { L. }\end{array}$ & & & $x$ & & 0.13 & - & - & Decoración fiestas navideñas. \\
\hline 3 & Calaguala & Phlebodium spp. & & $\mathrm{X}$ & & & 0.06 & - & - & Tratamiento para riñones. \\
\hline 4 & $\begin{array}{l}\text { Pino u } \\
\text { ocote }\end{array}$ & $\begin{array}{l}\text { Pinus oocarpa } \\
\text { Schiede ex Schltdl } \\
\text { y Pinus maximonoi } \\
\text { H.E. Moore. }\end{array}$ & $\mathrm{x}$ & & & & 0.63 & $\begin{array}{c}\text { Casi } \\
\text { Amenazado }\end{array}$ & - & $\begin{array}{l}\text { Madera para construcción, o leña. } \\
\text { Acículas empleadas en } \\
\text { elaboración de adobe. }\end{array}$ \\
\hline 5 & Orquídeas & Orchidaceae & & & $\mathrm{x}$ & & 0.06 & - & Apéndice II & Decoración de casas y jardines. \\
\hline 6 & $\begin{array}{l}\text { Flor de } \\
\text { izote }\end{array}$ & $\begin{array}{c}\text { Yucca guatemalensis } \\
\text { Baker }\end{array}$ & $\mathrm{x}$ & & & & 0.13 & - & - & Flores utilizadas como alimento. \\
\hline 7 & $\begin{array}{l}\text { Tuna } 0 \\
\text { Pitaya }\end{array}$ & Hylocereus spp. & $\mathrm{x}$ & & & $\mathrm{x}$ & 0.13 & - & Apéndice II & $\begin{array}{l}\text { El tallo y fruto es utilizado como } \\
\text { alimento, también para construir } \\
\text { cercos vivos. Considerado } \\
\text { problemática porque las espinas } \\
\text { lastiman o molestan al ganado. }\end{array}$ \\
\hline 8 & Cedro & Cedrela odorata L. & $\mathrm{x}$ & & & & 0.31 & Vulnerable & Apéndice III & Madera para construcción. \\
\hline 9 & Carbón & $\begin{array}{l}\text { Mimosa tenuiflora } \\
\text { (Willd.) Poir. }\end{array}$ & $\mathrm{x}$ & & & & 0.13 & - & - & $\begin{array}{l}\text { Fruto utilizado para alimentar el } \\
\text { ganado, madera como leña para } \\
\text { fogones. }\end{array}$ \\
\hline 10 & $\begin{array}{l}\text { Roble y } \\
\text { encino }\end{array}$ & Quercus spp. L. & $\mathrm{x}$ & & & & 0.75 & - & - & $\begin{array}{c}\text { Madera utilizada para leña, } \\
\text { elaboración de postes y } \\
\text { construcción. }\end{array}$ \\
\hline 11 & Quebracho & Lysiloma spp. Benth. & $\mathrm{x}$ & & & & 0.25 & - & - & $\begin{array}{c}\text { Elaboración de postes para cercos } \\
\text { y potreros, utilizados también para } \\
\text { leña. }\end{array}$ \\
\hline 12 & $\begin{array}{c}\text { Mora } \\
\text { silvestre }\end{array}$ & $\begin{array}{l}\text { Rubus ulmifolius } \\
\text { Schott. }\end{array}$ & $\mathrm{x}$ & & & & 0.12 & - & - & $\begin{array}{l}\text { Para consumo propio o para } \\
\text { comercio }\end{array}$ \\
\hline
\end{tabular}




\section{DISCUSIÓN}

El $90 \%$ de los pobladores se dedican a la agricultura y actividad ganadera, esto les permite frecuentar las áreas rurales y boscosas de su comunidad. Lo anterior, facilita la obtención de información a partir del conocimiento de las especies silvestres de fauna y flora de la zona. El $57 \%$ de las especies identificadas se reportaron en la categoría de problemáticas, sin embargo, esto pudo ser el resultado de la consulta en relación a los insectos dañinos.

Los entrevistados reportaron cuatro especies problemáticas de mamíferos, siendo el guazalo (Didelphis spp.) el de mayor importancia. Se mencionó al coyote (C. latrans), considerado en algunas zonas como problemático debido a su alta abundancia y depredación del ganado. Especies como el gato de monte (P. yagouaroundi) son reportadas como depredadores del ganado aviar en la zona y en otras publicaciones (Peña-Mondragón y Castillo, 2013, Peña-Mondragón, Castillo y Benítez-Malvido, 2014).

El venado cola blanca (O. virginianus) y el venado colorado (M. temama) son reportadas como una especie de cacería deportiva y de subsistencia. Según Morales-Mavil y Villa-Cañedo (1998), es bastante apetecible por los cazadores, debido al conocimiento de sus hábitos y los sitios que los animales frecuentan. Son empleados en otras regiones para crear artesanías para turismo o coleccionistas. Así mismo, se utilizan distintos órganos del venado cola blanca para tratar algunas patologías (Cortés-Gregorio et al., 2013; Hernández-López et al., 2012; Gil y Guiascón, 2012; García-Flores et al., 2014).

El mono cara blanca, está registrado por avistamientos directos en la zona (Pinel-Ramos, 2012), se encuentra sujeto a extracción del medio natural para mantenerlo como mascota en los hogares. Se considera necesario cuantificar esta actividad para generar estrategias de conservación, así como de ecoturismo para potenciar la atracción turística de la zona. En cuanto a la mención del puma "león de montaña" (P. concolor), a la fecha no se cuenta con un reporte de distribución de la especie en la zona, sin embargo, existe una alta probabilidad de que si se encuentre en la zona. En todo su rango de distribución sus poblaciones se han visto afectadas por la caza y pérdida de hábitat a causa de cambios de uso de suelo (Clavijo y Ramírez, 2009). Clavijo y Ramírez (2009) mencionan que esta especie es de importancia porque puede emplearse como especie sombrilla, contribuyendo a la conservación de otras especies. Dado lo anterior, su presencia en el AUMLB tendría grandes implicaciones 
en cuanto al manejo de la zona.

De los insectos, la gallina ciega presentó mayor IVU en el presente estudio, estas larvas son exclusivamente fitófagas, en términos económicos se les considera de los grupos más importantes porque afectan los cultivos (Borror et al., 1989). La única morfoespecie de molusco mencionada, fue la babosa, la cual afecta principalmente los cultivos de frijol, también es peligrosa para la salud humana al ser vector de un parásito que ataca los intestinos, especialmente en niños (Andrews et al., 1985). Se reportó que ambas afectan casi todos los cultivos que se encuentran en el AUMLB. Se observaron algunas especies de escarabajos estercoleros de la familia Scarabaeidae. Estas especies pueden ser empleadas como bioindicadores de la salud de ecosistema (Hanski y Camberfort, 1991; Estrada, 1998; Halffter, 2002).

En cuanto al reporte de las plantas utilizadas, en la categoría de uso medicinal se reportó a la calaguala (Phlebodium spp.) para el tratamiento de enfermedades renales, el mismo coincide con las propiedades medicinales descritas para este género en Honduras por Hernández-Cibrián y Nelson-Sutherland (2007). Se mencionó al cedro (C. odorata), como una especie maderable empleada en la categoría de consumo, de acuerdo con Mark y Rivers (2017) ésta es utilizada para una amplia gama de propósitos, incluyendo materia prima para muebles y artículos de artesanía, así como para reforestación y árbol de sombra. Se encuentra catalogado en el Apéndice III de CITES, y como Vulnerable en la Lista Roja de Especies Amenazadas de la UICN, por lo que se recomienda cuantificar la extracción y a partir de los resultados tomar acciones que regulen su uso.

El carbón (M. tenuiflora) categorizado como especie de consumo, es utilizado por los pobladores que se dedican a la ganadería, empleando los frutos en la alimentación del ganado. Cadena-Iñiguez y colaboradores (2014) mencionan que esta especie es ampliamente utilizada para ramoneo de ganado en México. También se registraron especies cuyos órganos vegetales eran introducidos de manera directa en las dietas alimenticias de los pobladores como el consumo de tallos y frutos de pitaya (Hylocereus spp.), frutos de mora silvestre (R. ulmifolius.) y flor de izote (Y. guatemalensis).

\section{CONCLUSIONES}

Con relación a la categoría de consumo, los mayores registros corresponden a extracción de fauna y flora silvestre. Se requiere una cuantificación de la extracción 
de estos recursos para estimar el impacto que dichas puedan tener en el ecosistema y en el caso de ser necesario tomar medidas de conservación. Por otro lado, se considera necesario realizar muestreos biológicos en la zona, tanto de fauna como de flora, para verificar la identificación taxonómica y su distribución dentro de la biosfera. Lo anterior, permitirá identificar zona de importancia para el uso, ecoturismo y conservación de las especies silvestres. Se recomienda a los comanejadores del AUMLB realizar una evaluación del impacto que la ganadería y los cultivos que podrían estar ejerciendo en las zonas boscosas, pues a través del recorrido realizado por Las Moras y El Picacho, se identificaron áreas donde se puede apreciar la perturbación que estas actividades ocasionan, por lo que se debe tener una consideración especial en este tema.

\section{AGRADECIMIENTOS}

Se agradece a las organizaciones comanejadoras del AUM La Botija, Enrich the world, la UMA de San Marcos de Colón, ICF Oficina Regional de San Marcos de Colon. Así mismo a la organización Sur en Acción y Ayuda en Acción por el apoyo logístico y técnico para la realización de la gira. Se agradece a los estudiantes del Curso de Manejo de Vida Silvestre del I periodo del 2018 de la Escuela de Biología que apoyaron la toma de datos en campo: Said Mejía, Lesdy Ordoñez, Augusto Salinas, Daniela Rivera, Carlos Sánchez y Sandra Ávila. También extendemos nuestro agradecimiento a Lilian Ferrufino, Germán Sandoval, Mario Solís y Franklin Castañeda por sus comentarios al documento.

\section{REFERENCIAS BIBLIOGRÁFICAS}

Andrews, K.L., y Dundee, D.S. (1987). Las babosas Veronicéllidos de Centroamérica con énfasis en Sarasinula plebeia (= Vaginulus plebeius) ${ }^{\star}$. Ceiba, 28(2): 163-172.

Andrews, K.L., Valverde, V. II, y Ramírez, O. (1985). Preferencia alimenticia de la babosa, Sarasinula plebeia (Fisher). Ceiba, 26(1): 59-65.

Barrera-Bassols, N. y Toledo V.M. (2005). Ethnoecology of the Yucatec Maya: Symbolism, knowledge and management of the natural resources. Journal of Latin American Geography, 4(1): 9-41.

Biurrun, E., Galetto, L., Anton, A. M., y Biurrun, F. (2007). Plantas silvestres comestibles utilizadas en poblaciones rurales de la Provincia de La Rioja (Argentina). Volumen espe- 
cial de Etnobotánica, 121-140.

Borror, D., Triplehorn, N., y Johnson, N. (1989). An introduction to the study of insects (6 ed.). Sauders college publishing.

Caballero, R., Thomé, J.W., Andrews, K.L., y Rueda, A. (1991). Babosas de Honduras (Soleolifera: Veronicellidae): Biología, Ecología, Distribución, Descripción, Importancia Económica, y Claves para su Identificación. Ceiba, 32(2): 107-126.

Cadena-Iñiguez, P., Cruz-Morales, F.D.C., y Ballinas-Albores, E. (2014). Tepezcohuite (Mimosa tenuiflora (L) Wild) El Árbol de la Piel. Agroproductividad, 7(6): 10-16.

Castañeda-Sifuentes, R., y Albán-Castillo, J. (2016). Importancia cultural de la flora silvestre del distrito de Pamparomas, Ancash, Perú. Ecología Aplicada, 15(2): 151-169.

Challenger, A. (1998). Utilización y conservación de los ecosistemas terrestres de México. Pasado, presente y futuro de México. México. CONABIO/ UNAM/ASM, 847.

Clavijo, A., y Ramírez, G.F. (2009). Taxonomía, distribución y estado de conservación de los felinos suramericanos: revisión monográfica. Bol. cient. mus. hist. nat., 13(2):43-60.

Cortés-Gregorio, I., Pascual-Ramos, E., Medina-Torres, S. M., Sandoval-Forero, E. A., Lara-Ponce, E., Piña-Ruíz, H. H., Martínez-Ruíz, HH., y Rojo-Martínez, G. E. (2013). Etnozoología del pueblo mayo-yoreme en el norte de Sinaloa: uso de vertebrados silvestres. Agricultura, sociedad y desarrollo, 10(3): 335-358.

Cruz, F. (2005). Miasis (1 ed.). México: Universidad Nacional Autónoma De México Facultad de medicina veterinaria y zootecnia.

De la Ossa, A., y De la Ossa, V.J. (2012). Índice de valor de uso para fauna silvestre en la región del San Jorge, Mojana Sucreña, Colombia. Revista Colombiana de Ciencia Animal - RECIA, 4(2): 308-319.

Escoto, N. (2004). El cultivo del frijol. Tegucigalpa, Honduras: Secretaría de Agricultura y Ganadería y Dirección de Ciencias y Tecnología Agropecuaria.

Estrada, A. (2015). Clase Arachnida Orden Ixodida: Las Garrapatas (13 ed.). Ibero Diversidad Entomológica.

Estrada, A. C. (1998). Dung and carrion beteles intropical rain forest and agricultural habitats at Los Tuxtlas. Tropical Ecology, 14 (5): 577-593.

García-Flores, A., Lozano-García, M. A., Ortíz-Villaseñor, A. L., y Monroy-Martinez, R. (2014). Uso de Mamíferos Silvestres por habitantes del Parque Nacional El Tepozteco, Morelos, México. Etnobiología, 12(3): 57-67.

Geilfus, F. (1997). 80 herramientas para el desarrollo participativo: diagnóstico, planificación, monitoreo y evaluación. IICA / GTZ, 208.

Gil, R. A., y Guiascón, O. G. (2012). Uso de la Fauna Silvestre en la Comunidad Maya Villa de Guadalupe, Campeche, México. Etnobiología, 10 (2) 1-11.

Goodman, L. A. (1961). Snowball Sampling. Annals of Mathematical Statistics, 148-170.

González-Bocanegra, K., Romero-Berny, E.I., Escobar-Ocampo, M.C., y García-Del Valle, Y. (2011). Aprovechamiento de fauna silvestre por comunidades rurales en los humedales de Catazajá - La Libertad, Chiapas, México. Ra Ximhai, 7(2): 219-230.

Halffter, G., y Arellano, L. (2002). Response of dung beetle diversity to human-induced changes in a tropical landscape bioTropica, 34 (1): 144-154. 
Hanski I., y Camberfort, I. (1991). Competition in dung beetles. University Press, Princeton, 1: 305-329.

Hernández-Cibrián, R. K., y Nelson-Sutherland, C. H. (2007). Etnobotánica de los Helechos de Honduras. Ceiba, 48: 1-10.

Hernández-López, A., López-Alamilla, E., Ramírez, A. R., y Aquino-Bravata, V. (2012). Diagnóstico del Uso de la Fauna Silvestre, en el Área de Protección de Flora y Fauna "Cañón del Usumacinta" Tenosique, Tabasco. Ra Ximhai, 1-13.

ICF (Instituto de Conservación Forestal). (2009). Plan de Manejo Área de Usos Múltiples Montaña La Botija. 63 pp.

Ladio, A., y Lozada, M. (2004). Patterns of use and knowledge of wild edible plants in distinct ecological environments: a case study of mapuche community from northwestern Patagonia. Biodiversity \& Conservation, 13(6): 1153-1173.

Ladio, A., y Rapoport, E. (1999). El Uso de Plantas Silvestres Comestibles en una Población Suburbana del Noreste de la Patagonia. Parodiana, 11 (1-2): 49-62.

Mark, J. y Rivers, M.C. (2017). Cedrela odorata. The IUCN Red List of Threatened Species 2017: e.T32292A68080590. http://dx.doi.org/10.2305/IUCN.UK.2017-3.RLTS.T32292A 68080590. Recuperado de la página web 02 de noviembre de 2018.

Miranda, A. (1993). Manejo de fauna silvestre. Ciencias, (7): 103-110.

Monterubio, C.L., Cruz Lara, L.E., Naranjo Piñera, E.J., y Barragán Torres, F. (2007). Uso y Conservación de Mamíferos Silvestres en una Comunidad De Las Cañadas de la Selva Lacandona, Chiapas, México. Etnobiología, 5(1): 99-107.

Morales Mávil, J.E., y Villa Cañedo, J.T. (1998). Notas Sobre el Uso de la Fauna Silvestre en Catemaco, Veracruz, México. Acta Zoológica Mexicana, 73(73): 127-143.

Morón Ríos, M. A. (1986). El género Phyllophaga en México. Morfología, distribución y sistemática supraespecífica (Insecta: Coleoptera). Instituto de Ecología, México, p.314.

Ochoa, J., Ladio, A., y Lozada, M. (2010). Uso de recursos herbolarios entre mapuches y criollos de la comunidad campesina de Arroyo Las Minas (Río Negro, Patagonia, Argentina). Boletín Latinoamericano y del Caribe de Plantas Medicinales y Aromáticas, 9(4): 99-107.

Ojasti, J. (1993). Utilización de la fauna silvestre en América Latina: situación y perspectivas para un manejo sostenible. Roma, Italia.

Otzen, T., y Manterola, C. (2017). Técnicas de muestreo sobre una población a estudio. Int. J. Morphol., 35(1): 227-232.

Ozonas, L., y Pérez, A. (2005). La entrevista semiestructurada. Notas sobre una práctica metodológica desde una perspectiva de género. La Aljaba, 9: 198-2003.

Peña-Mondragón, J. L., y Castillo, A. (2013). Depredación de ganado por jaguar y otros carnívoros en el noreste de México. Therya, 4(3): 431-446.

Peña-Mondragón, J. L., Castillo, A., y Benítez-Malvido, J. (2014). Primer registro de Coyote (Canis latrans) en la Región de La Selva Lacandona, Chiapas, México. Acta Zoológica Mexicana, 30(3): 696-700.

Pérez, E., y Ojasti, J. (1996). La utilización de la fauna silvestre en la América tropical y recomendaciones para su manejo sustentable en las sabanas. Ecotropicos, 9(2): 71-82. 
Pinel-Ramos, E.J. (2012). Presencia y densidad poblacional de Cebus capucinus en la Montaña de El Ojochal, dentro del Área Protegida de Usos Múltiples "La Botija", San Marcos de Colón, Choluteca. Tesis de Grado. UNAH, CU. 175 p.

Potter, D. A. (1998). Destructive turfgrass insects. Biology, diagnosis and control. Chelsea, Michigan, USA: An Arbor Press. 368 p.

SENASICA-DGSV. (2016). Langosta Centroamericana Schistocerca piceifrons piceifrons (Walker, 1870) (Orthoptera: Acrididae). Servicio Nacional de Sanidad, Inocuidad y Calidad Agroalimentaria -Dirección General de Sanidad Vegetal- Centro Nacional de Referencia Fitosanitaria- Grupo Especialista Fitosanitario. Ficha Técnica. Tecámac, México $18 \mathrm{p}$.

Tamayo, G. (2001). Diseños muestreales en la investigación. Semestre Económico, 4(7): 1-14.

TCA (Tratado de Cooperación Amazónica). (1995). Uso y conservación de fauna silvestre en la Amazonía. Secretaría Pro-Tempore, Lima, 216 p.

UNESCO (United Nations Educational, Scientific and Cultural Organization). (2017). Boletín UNESCO San José Junio-Julio 2017. 9 pp. En línea, recuperado el 17-IX-18, URL: http://www.unesco.org/new/fileadmin/MULTIMEDIA/FIELD/San-Jose/images/Boletin_SJO_Julio_2017.pdf 